Euskal ikerketen aldizkaria | Revue d'études basques |

Revista de estudios vascos | Basque studies review

$13 \mid 2009$

Numéro XIII

\title{
Tester un calque grammatical grâce à la méthode de la covariation d'abstrat
}

\section{Michel Etchebarne}

\section{OpenEdition \\ Journals}

Édition électronique

URL : http://journals.openedition.org/lapurdum/2029

DOI : 10.4000/lapurdum.2029

ISSN : 1965-0655

\section{Éditeur}

IKER

\section{Édition imprimée}

Date de publication : 1 février 2009

Pagination : 113-126

ISBN : 978-2-86781-409-X

ISSN : $1273-3830$

Référence électronique

Michel Etchebarne, «Tester un calque grammatical grâce à la méthode de la covariation d'abstrat », Lapurdum [En ligne], 13 | 2009, mis en ligne le 15 avril 2013, consulté le 21 avril 2019. URL : http:// journals.openedition.org/lapurdum/2029; DOI : 10.4000/lapurdum.2029 


\title{
Tester un calque grammatical grâce à la méthode de la covariation d'abstrat
}

\author{
Michel ETCHEBARNE \\ Université Paris IV-Sorbonne
}

\section{Laburpena:}

La présente étude propose une méthode qui permet, à certaines conditions et dans certaines limites, de tester une hypothèse de calque grammatical. La méthode ne permet pas de donner une preuve de calque mais fournit un faisceau de présomptions, plus ou moins précises et concordantes, de calque. L'étude se limite à l'état de langue synchronique contemporain et n'aborde qu'accessoirement la dimension diachronique.

\section{Abstract:}

This paper proposes a method which allows, under certain conditions and within certain limits, to test a contact-induced grammatical change hypothesis. The method does not claim to prove such a change, but it gives a good presumption of it. The work restricts itself to today's state of language and only incidentally approaches the diachronic dimension.

Gako hitzak: Tester une hypothèse de calque grammatical

Keywords: Testing a contact-induced grammatical change hypothesis

La recherche sur les calques grammaticaux est traditionnellement un domaine d'études où les consensus se forment difficilement. La difficulté réside moins sur la possibilité même du calque (la majorité des spécialistes en admettent le principe), que sur une question de preuve. En effet, lorsqu'une langue calque une construction grammaticale sur le modèle d'une autre langue, elle imite une construction étrangère en recourant à des formes matérielles qui lui sont propres et en imprimant une partie de sa propre logique dans la construction calquée. De sorte que cette dernière ne peut que rarement être vue comme la transposition pure et simple de la construction de la langue source dans la langue cible. De plus, les mêmes schémas de grammaticalisation tendent à se reproduire dans les langues les plus diverses, de même que dans les divers états de langue successifs d'une même langue. On peut donc aussi bien rendre compte d'une ressemblance entre deux langues par une convergence fortuite que par un calque. Dès lors, il est rare qu'un diagnostic de calque fasse l'unanimité. On touche là à l'une des difficultés épistémologiques radicales de la linguistique de contact. Pourtant, la façon dont le dossier est régulièrement refermé puis rouvert, et le caractère vivace de ces problématiques semblent témoigner de ce que ces recherches ont un intérêt pour la 
connaissance des langues et de leur évolutivité. C'est sous cet angle que la question des calques peut être intéressante : que peut nous apprendre sur une langue donnée la façon dont celle-ci imite, ou n'imite pas, certains aspects d'une autre langue?

La présente étude propose une méthode qui permet, à certaines conditions et dans certaines limites, de tester une hypothèse de calque. La méthode ne permet pas de donner une preuve de calque mais fournit, comme disent les juristes, un faisceau de présomptions, plus ou moins précises et concordantes, de calque. L'étude se limite à l'état de langue synchronique contemporain et n'aborde qu'accessoirement la dimension diachronique. Elle examine quatre hypothèses de calque déjà connues mais qui, à ma connaissance, n'ont pas été testées sur la base de protocoles conçus à cet effet.

Plan de la note :

0 . Présentation de la méthode

1. Parfaits romans

2. Izan / egon $=$ ser $/$ estar

3. ${ }^{*}$ Edun $/$ eduki $=$ haber $/$ tener

4. Constructions obliques

\section{Présentation de la méthode}

\section{Carte 1}

\section{Covariation d'adstrat : le principe}

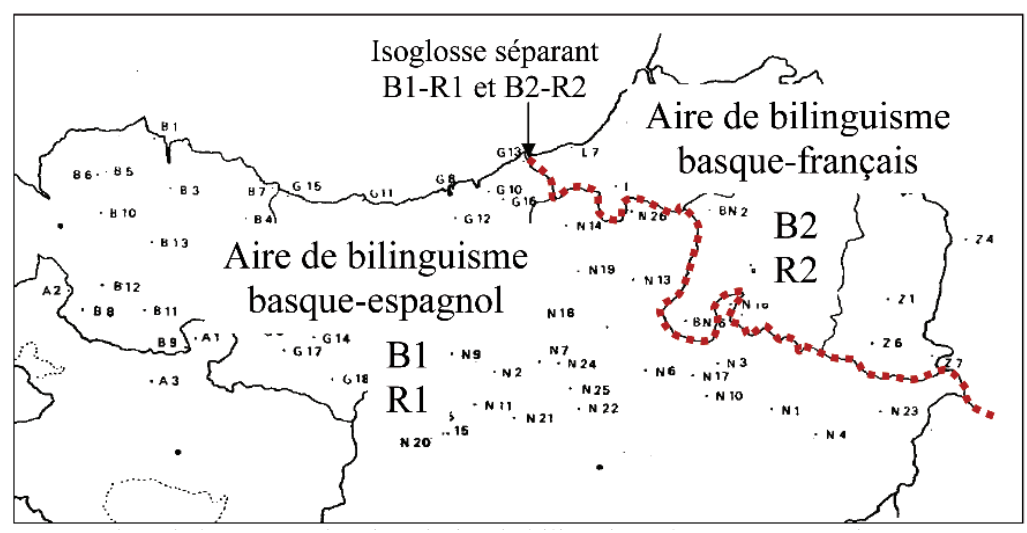

B1 = variété de basque parlée dans l'aire de bilinguisme basque-espagnol

R1 = variété de roman parlée dans l'aire de bilinguisme basque-espagnol

B2 = variété de basque parlée dans l'aire de bilinguisme basque-français

R2 = variété de roman parlée dans l'aire de bilinguisme basque-français

Le fait de caractériser deux variétés de basque par rapport à une isoglosse séparant le domaine hispano-roman du domaine gallo-roman ne se justifie que pour les besoins de notre étude dans le cas présent. On pourrait subdiviser des variétés de basque en utilisant d'autres isoglosses pour les besoins d'autres objectifs de recherche. 
Le principe de la méthode est le suivant.

Lorsque l'on constate une différence grammaticale entre B1 et B2, et que cette différence peut être mise en correspondance avec une différence grammaticale entre Rl et R2, alors il existe une présomption $\square$ et seulement une présomption $\square$ de calque ${ }^{1}$.

Comparons les deux phrases suivantes (carte 2):

(1) atzo žin da gizun bat (Alçay/Altzai, Soule, EAEL ${ }_{236}$ )

«un homme est venu hier

$\begin{array}{lllll}\text { atzo } & \text { jin } & \text { da } & \text { gizon } & \text { bat } \\ \text { hier } & \text { venu } & \text { il/est } & \text { homme } & \text { un }\end{array}$

(2) atzo etorri zen gizon bat (Hondarribia, Guipuscoa, $E A E L_{236}$ )

«ayer vino un hombre »

$\begin{array}{lllll}\text { atzo } & \text { etorri } & \text { zen } & \text { gizon bat } \\ \text { ayer } & \text { venido } & \text { él/era } & \text { hombre } & \text { un }\end{array}$

Carte 2

Auxiliaire au passé / auxiliaire au présent

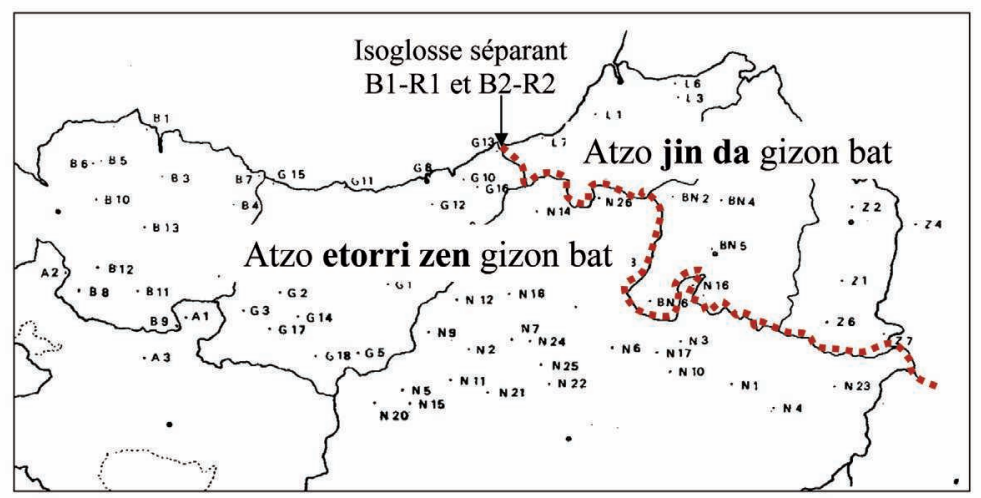

En B1, la quasi-totalité des informateurs ont utilisé l'auxiliaire au passé (zen « il était ») alors qu'en B2 tous les informateurs ont utilisé l'auxiliaire au présent ( $d a$ « il est ») $)^{3}$. Or, on constate que

1. On pourrait, en droit, considérer que c'est R1 ou R2 qui covarient avec B1 ou B2. C'est le contexte sociologique local (phénomènes de diglossie, etc.) qui va orienter le diagnostic relatif au sens dans lequel le calque a pu s'opérer. D'autre part, dans les cas antérieurs à la période moderne, l'influence est rapportable aux variétés romanes circum-pyrénéennes (gascon, béarnais, aragonais, etc.) et non pas aux français et espagnol modernes, dont l'action ne peut être qu'assez récente. Il importe néanmoins de remarquer que, à côté de leurs caractéristiques propres, les variétés romanes circum-pyrénéennes modernes et médiévales participent, au même titre que le français et l'espagnol, des macroévolutions panromanes.

2. Les phrases libellées en français et en espagnol figurant entre guillemets sont celles que les enquêteurs ont demandé aux informateurs de traduire dans leur parler basque. 
Rl utilise dans ce contexte le perfecto simple (l'équivalent du passé simple français : vino « il vint »), et que R2 utilise le passé composé (participe passé + auxiliaire au présent). On est ici en présence d'une présomption et seulement une présomption de calque.

La méthode comporte trois temps:

(i) comprendre, d'une part, la différence entre B1 et B2 ; et, d'autre part, entre R1 et R2 ;

(ii) vérifier dans des corpus non traduits que la différence entre B1 et B2 préexiste au corpus qui est à l'origine du constat de covariation ${ }^{4}$;

(iii) tester auprès des informateurs de B1 la construction utilisée par les informateurs de B2 et inversement.

Linvestigation diachronique n'est pas abordée à ce stade. Il est en effet préférable de disjoindre l'investigation synchronique de l'investigation diachronique, d'une part afin d'éviter les phénomènes d'interprétation anticipée (même si rien n'interdit, bien entendu, de faire des hypothèses dès le départ) ; et, d'autre part, parce que l'investigation synchronique vaut pour elle-même : menée de façon rigoureuse, elle ne peut être contredite par l'investigation diachronique.

Reprenons les trois temps de l'approche synchronique.

(i) Différence entre R1 et R2 ${ }^{5}$. Lespagnol et le français disposent chacun de deux paradigmes pour dénoter un événement achevé survenu dans le passé. En simplifiant, on peut dire que le perfecto simple est au passé simple ce que le perfecto compuesto est au passé composé.

En espagnol standard péninsulaire, le choix entre perfecto simple et perfecto compuesto se fait, dans le contexte d'une phrase telle que (2), de la façon suivante ${ }^{6}$.

Cas A : lorsqu'un événement est considéré comme achevé à l'intérieur d'une période de référence elle-même achevée au moment de l'énonciation, on utilise le perfecto simple ${ }^{7}$.

Cas B : lorsqu'un événement est considéré comme achevé à l'intérieur d'une période de référence non achevée au moment de l'énonciation, on utilise le perfecto compuesto.

Dans une phrase telle que ayer vino un hombre, le moment de l'énonciation ne peut être qu'aujourd'hui, et la période de référence à l'intérieur de laquelle s'est produit l'événement (ayer, vino) est achevée au moment de l'énonciation. On utilise donc le perfecto simple.

3. Deux informateurs de Bl, situés en Biscaye septentrionale, ont utilisé l'auxiliaire au présent (Bermeo et Mungia). Il faudrait évaluer la portée de ces relevés par enquête (cf. infra).

4. Dans l'atlas EAEL, des phrases cibles ont été fournies par les informateurs à partir de phrases sources formulées en espagnol et en français. Un tel protocole peut provoquer des contrastes qui ne sont pas à interpréter comme des contrastes dialectaux mais comme un simple reflet des écarts entre les deux phrases sources.

5. Je n'aborde pas la différence entre B1 et B2 car celle-ci devrait faire l'objet d'enquêtes interdialectales spécifiques.

6. Les variétés R1 et R2 susceptibles d'avoir exercé une influence sur B1 et B2 sont celles qui sont effectivement utilisées dans les zones de bilinguisme précitées. On suppose ici que ces variétés sont les variétés standards correspondantes.

7. Se emplea el perfecto simple con los adverbios que indican que la acción se produce en un período de tiempo en el que no está incluido el momento presente del que habla : ayer, anoche, el mes pasado [...] (Alarcos Llorach, 1994 [1970] : 25). 


\begin{tabular}{|c|c|c|}
\hline \multicolumn{3}{|c|}{ Règle applicable en R1 } \\
\hline \multicolumn{2}{|c|}{ Cas A } & Cas B \\
\hline $\begin{array}{l}\text { Période de } \\
\text { référence } \\
\text { ayer, anoche, } \\
\text { el otro día, } \\
\text { la semana pasada, } \\
\text { el mes pasado, } \\
\text { el año pasado, etc. }\end{array}$ & $\begin{array}{l}\text { Moment de } \\
\text { l'énonciation }\end{array}$ & $\begin{array}{c}\text { Période de référence } \\
\text { Moment de l'énonciation } \\
\text { hoy, } \\
\text { esta noche, } \\
\text { esta semana, } \\
\text { este mes, } \\
\text { este año, etc. } \\
\text { X }\end{array}$ \\
\hline $\mathrm{X}$ & $\mathrm{Y}$ & $\mathrm{Y}$ \\
\hline Perfecto simple & & Perfecto compuesto \\
\hline $\begin{array}{l}\mathrm{X}=\text { événe } \\
\mathrm{Y}=\text { mome }\end{array}$ & $\begin{array}{l}\text { nt achevé d } \\
\text { de l'énoncia }\end{array}$ & ode de référence \\
\hline
\end{tabular}

Le français oral ayant perdu l'usage du passé simple, passé simple et passé composé ne sont plus en distribution complémentaire ${ }^{8}$. La seule façon de dénoter le type d'événement précité consiste à utiliser le passé composé et, donc, un auxiliaire au présent.

(ii) Vérification dans des corpus non traduits que la différence entre B1 et B2 préexiste à l'enquête. La vérification confirme que la différence entre B1 et B2 préexiste à l'enquête.

(iii) Jugement d'acceptabilité. J'ai demandé à des informateurs de Soule (aire B2-R2), qui fournissent eux-mêmes une réponse du type (1), de se prononcer sur la phrase basque fournie en B1. Le jugement a été le suivant :

\begin{tabular}{|c|c|c|c|c|}
\hline $\begin{array}{c}\text { Dans votre usage personnel de langue, } \\
\text { est-il possible de dire: }\end{array}$ & Oui & $\begin{array}{c}\text { Plutôt } \\
\text { oui }\end{array}$ & $\begin{array}{c}\text { Plutôt } \\
\text { non }\end{array}$ & Non \\
\hline $\begin{array}{c}\text { atzo jin zen gizon bat } \\
\text { «un homme est venu hier» }\end{array}$ & $\mathrm{X}$ & & & \\
\hline
\end{tabular}

Les informateurs considèrent que l'on peut utiliser l'auxiliaire au présent comme au passé dans le contexte précité ${ }^{10}$. D'autre part, d'après mes informations, si l'on testait la phrase B2 dans l'aire B1, on obtiendrait la réponse suivante ${ }^{11}$ :

8. La déperdition a débuté au $16^{e}$ siècle et de façon variable selon les régions. Elle est déjà assez bien établie en certains endroits au $17^{\text {e }}$. Le mouvement s'est accéléré au $19^{\mathrm{e}}$ siècle et est arrivé à terme au $20^{e}$ (Brunot \& Bruneau, 1969: 334-335, 348).

9. Ce jugement d'acceptabilité devrait être testé dans toute l'aire B2.

10. Prédiction à valider par enquête. Il est possible que la prédiction ne se vérifie pas partout de façon uniforme en Bl (cf. notamment le cas de la Biscaye septentrionale).

11. Prédiction à valider par enquête. Il est possible que la prédiction ne se vérifie pas partout de façon uniforme en Bl (cf. notamment le cas de la Biscaye septentrionale). 


\begin{tabular}{|c|c|c|c|c|}
\hline $\begin{array}{c}\text { Dans votre usage personnel de langue, } \\
\text { est-il possible de dire: }\end{array}$ & Oui & $\begin{array}{c}\text { Plutôt } \\
\text { oui }\end{array}$ & $\begin{array}{c}\text { Plutôt } \\
\text { non }\end{array}$ & Non \\
\hline $\begin{array}{c}\text { atzo etorri da gizon bat } \\
\text { «ayer vino un hombre» }\end{array}$ & & & & $\mathrm{X}$ \\
\hline
\end{tabular}

En résumé, on constate les faits suivants dans le contexte de la phrase testée : (i) l'espagnol utilise le perfecto simple (c'est-à-dire un temps de passé) et le français le passé composé (c'est-à-dire un syntagme d'aspect perfectif rattachable au présent du fait du temps de l'auxiliaire) ; (ii) le contraste dialectal entre B1 et B2 préexiste à l'enquête qui est à l'origine du constat de variation ; (iii) il existerait une asymétrie dans l'usage des temps entre informateurs de B2 (qui pourraient utiliser l'auxiliaire au présent et au passé, avec des nuances qu'il faudrait évaluer par enquête), et informateurs de B1 (qui ne pourraient utiliser, dans ce contexte, que l'auxiliaire au passé).

La question qui se pose à ce stade est la suivante : les choses étant ce qu'elles sont et sous réserve de contrôles à réaliser par voie d'enquêtes complémentaires, faudrait-il supposer que le contraste dialectal entre B1 et B2 est en partie conditionné par le contraste dialectal entre R1 et R2 ?

Ce qui plaiderait dans ce sens, dans le contexte de la phrase précitée, c'est le fait que l'isoglosse qui sépare la variété de basque où l'on ne peut utiliser l'auxiliaire qu'au passé (B1) de la variété de basque où l'on peut utiliser l'auxiliaire au présent et au passé (B2), se superpose à l'isoglosse qui sépare la variété de roman où l'on ne peut utiliser que le perfecto simple, c'est-à-dire un temps de passé (R1), de la variété de roman où l'on ne peut utiliser que le passé composé, c'est-à-dire un syntagme d'aspect perfectif rattachable au présent.

Dans le contexte de langue précité, la règle basque $\mathrm{Bl}$ semble être à la règle romane $\mathrm{R} l$ ce que la règle basque $\mathrm{B} 2$ semble être à la règle romane $\mathrm{R} 2$. Ceci conduit à la prédiction suivante, qu'il faudrait tester en B1:

Quant à B2, la règle correspondante serait que l'auxiliaire peut être fléchi au présent ou au passé

\begin{tabular}{|c|c|c|}
\hline \multicolumn{3}{|c|}{ Règle postulable (mais à tester) en B1 } \\
\hline \multicolumn{2}{|c|}{ Cas A } & Cas B \\
\hline $\begin{array}{c}\text { Période de } \\
\text { référence } \\
\text { atzo, } \\
\text { bart arratsean, } \\
\text { lehengo egunean, } \\
\text { joan den (aurreko) } \\
\text { astean } \\
\text { hilabetan iaz etc }\end{array}$ & $\begin{array}{l}\text { Moment de } \\
\text { l'énonciation }\end{array}$ & $\begin{array}{c}\text { Période de référence } \\
\text { Moment de l'énonciation } \\
\text { gaur, } \\
\text { bart, } \\
\text { aste honetan, } \\
\text { hilabete honetan, } \\
\text { aurten, etc. }\end{array}$ \\
\hline $\begin{array}{l}\text { hilabetan, iaz, etc. } \\
\text { X }\end{array}$ & Y & $\begin{array}{l}\mathrm{X} \\
\mathrm{Y}\end{array}$ \\
\hline $\begin{array}{c}\text { etorri zen } \\
\text { (aux. au passé) }\end{array}$ & & etorri da (auxiliare au présent \\
\hline $\begin{array}{l}\mathrm{X}=\text { événe } \\
\mathrm{Y}=\text { mome }\end{array}$ & $\begin{array}{l}\text { hent achevé da } \\
\text { it de l'énonciat }\end{array}$ & iode de référence \\
\hline
\end{tabular}


dans le cas A (avec des nuances qui seraient aussi à tester par voie d'enquête).

Lanalyse synchronique des données ne permet pas en revanche de dire dans quel sens la covariation a eu lieu : seulement de B1 sur le modèle de R1 ; seulement de B2 sur le modèle de R2 ; à la fois de $\mathrm{B} 1$ et de $\mathrm{B} 2$ sur le modèle respectivement de $\mathrm{R} 1$ et $\mathrm{R} 2^{12}$.

\section{Izan / egon = ser / estar}

Observons les phrases et la carte suivantes:

(3) atzo etorri zan gizona gaxo zegon (Saint-Sébastien/Donostia, G, EAEL ${ }_{266}$ ) «el hombre que vino ayer estaba enfermo»

$\begin{array}{llllll}\text { atzo } & \text { etorri } & \text { zen } & \text { gizona } & \text { gaixo } & \text { zegoen } \\ \text { ayer } & \text { venido } & \text { que/era } & \text { el/hombre } & \text { enfermo } & \text { estaba }\end{array}$

(4) etxea txikiya da (Saint-Sébastien/Donostia, G, EAEL ${ }_{244}$ )

«la casa es pequeña»

$\begin{array}{lll}\text { etxea } & \text { txikia } & \text { da } \\ \text { la/casa } & \text { pequeña } & \text { es }\end{array}$

(5) atzo yin den gizona eri zen (Itxassou/Itsasu, Labourd, EAEL ${ }_{266}$ ) «l'homme qui est venu hier était malade »

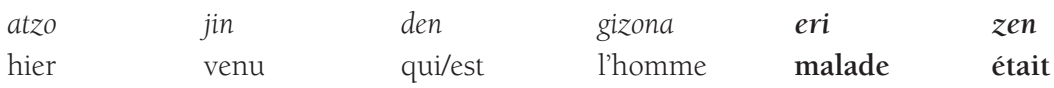

(6) etxea ttipia da (Itxassou/Itsasu, L, EAEL ${ }_{244}$ )

«la maison est petite »

etxea tipia da

la/maison petite est

12. Liçarrague utilise l'auxiliaire au présent avec atzo, dans des formes relatives, à deux reprises (Actes des apôtres 7, 28 et Lettre aux Hébreux 13,8) alors que le texte source du 16 siècle comporte, en regard, un passé simple et un passé composé. En français, avec hier, le passé simple prédomine largement dans les écrits du $17^{\mathrm{e}}$ siècle (98\% de passés simples contre $2 \%$ de passés composés). Le basculement se produit brusquement au 18 e siècle (48\% de passés simples contre $52 \%$ de passés composés) sans que l'on sache exactement pourquoi (Caron \& Liu, 1999). Quoi qu'il en soit, quelles que soient les tendances révélées par les textes, tant dans le domaine roman que basque, il importe, comme on l'a dit, avant de confronter le diagnostic de covariation avec les textes du passé, de cerner les contrastes dialectaux basques, pour eux-mêmes, dans la synchronie contemporaine. 


\section{Carte 3}

Izan $/$ egon $=$ ser $/$ estar $\left(\mathrm{EAEL}_{266}\right)$

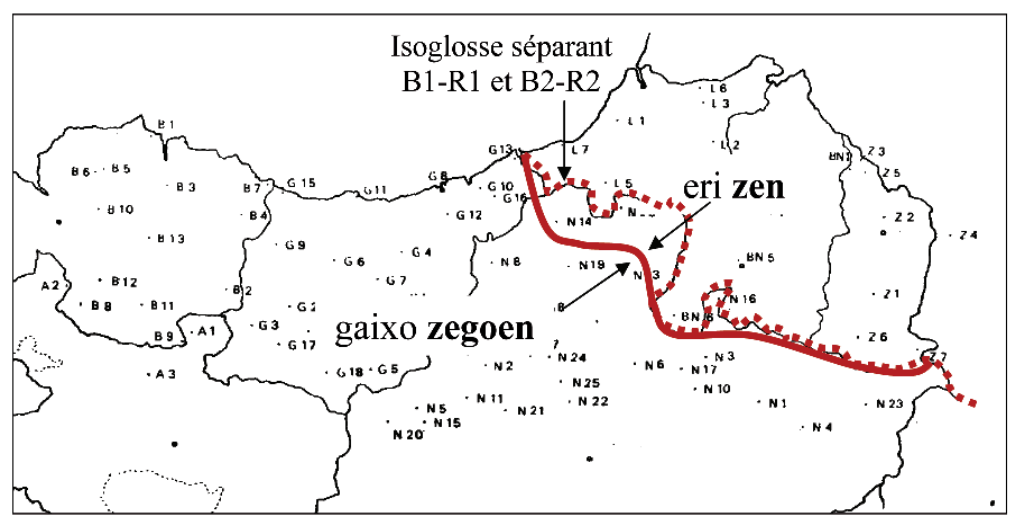

Dans le contexte des phrases (3) et (5), on constate, que la quasi-totalité des informateurs de B1 ont utilisé egon « estar » alors que les informateurs de B2 ont utilisé izan «être ». Lisoglosse séparant le domaine où egon se substitue à izan ne coïncide pas parfaitement avec la limite entre R1 et R2 mais elle en est très proche. On est donc en présence d'une présomption de calque. Reprenons les trois étapes précitées.

(i) Différence entre R1 et R2. Alors que le français ne dispose que d'un seul verbe être, l'espagnol dispose de deux verbes, ser et estar, le premier dénotant une propriété inhérente au référent, le second une caractéristique passagère de celui-ci.

(ii) Vérification dans des corpus non traduits que la différence entre B1 et B2 préexiste à l'enquête. La vérification confirme que la différence entre B1 et B2 préexiste à l'enquête.

(iii) Jugement d'acceptabilité. J'ai demandé à des informateurs de Soule (aire B2-R2), qui fournissent eux-mêmes une réponse du type (5), de se prononcer sur la phrase basque fournie en B1. Le jugement a été le suivant :

\begin{tabular}{|c|c|c|c|c|}
\hline $\begin{array}{c}\text { Dans votre usage personnel de langue, est-il possible } \\
\text { de dire, avec le sens de la phrase source : }\end{array}$ & Oui & $\begin{array}{c}\text { Plutôt } \\
\text { oui }\end{array}$ & $\begin{array}{c}\text { Plutôt } \\
\text { non }\end{array}$ & Non \\
\hline $\begin{array}{c}\text { atzo jin zen gizona eri zagon }{ }^{13} \\
\text { «l'homme qui est venu hier était malade» }\end{array}$ & & & & $\mathrm{X}$ \\
\hline
\end{tabular}

13. Le verbe egon synthétique au passé n'est pas utilisé par les informateurs, mais egoiten zen n'est pas non plus jugé possible dans le contexte de la phrase source.

14. Prédiction à valider par enquête (la maladie est envisagée ici dans sa forme passagère). 
D'autre part, d'après mes informations, si l'on testait la phrase B2 dans l'aire B1, on obtiendrait la réponse suivante ${ }^{14}$ :

\begin{tabular}{|c|c|c|c|c|}
\hline $\begin{array}{c}\text { Dans votre usage personnel de langue, est-il possible } \\
\text { de dire, avec le sens de la phrase source }\end{array}$ & Oui & $\begin{array}{c}\text { Plutôt } \\
\text { oui }\end{array}$ & $\begin{array}{c}\text { Plutôt } \\
\text { non }\end{array}$ & Non \\
\hline $\begin{array}{c}\text { atzo etorri zen gizona gaixo zen } \\
\text { «el hombre que vino ayer estaba enfermo }\end{array}$ & & & & $\mathrm{X}$ \\
\hline
\end{tabular}

En résumé, le parcours suivi amène à diagnostiquer que le contraste dialectal entre B1 et B2 est en partie conditionné par le contraste dialectal entre R1 et R2. Les informateurs de B1 tendent à transposer dans leur langue l'opposition de l'espagnol ser / estar. Pour cela, il a été recouru au verbe autochtone egon dont l'une des acceptions primaires est « rester », verbe qui a été mis en opposition avec le verbe izan « être ». Alors que egon est utilisé (cf. espagnol estar) lorsqu'il s'agit de dénoter une caractéristique passagère du référent comme en (3), les mêmes informateurs utilisent izan (cf. espagnol ser) comme copule d'un attribut inhérent au référent comme en (4). Les locuteurs de B2 utilisent quant à eux izan « être » dans les deux cas.

\section{3. ${ }^{*}$ Edun / eduki $=$ haber $/$ tener}

Observons les phrases et la carte suivantes:

(7) ez ditue kutxillook eraman (Zegama, G, EAEL ${ }_{251}$ )

«no se han llevado los cuchillos»

$\begin{array}{llll}\text { ez } & \text { dituzte } & \text { kutxilloak } & \text { eraman } \\ \text { no } & \text { ellos/los/han l } & \text { os/cuchillos } & \text { levado }\end{array}$

(8) zenbat idi dauzkee errin (Zegama, $G, E A E L_{262}$ )

«icuántos bueyes tienen en el pueblo?»

$\begin{array}{llll}\text { zenbat } & \text { idi } & \text { dauzkae } & \text { herrian } \\ \text { cuánto } & \text { buey } & \text { ellos/los/tienen } & \text { en/el/pueblo }\end{array}$

(9) ez dituzte beren kanitak hartu (Bidarray/Bidarrai, Basse-Navarre, EAEL ${ }_{251}$ )

«ils n'ont pas emporté leurs couteaux»

$\begin{array}{lllll}\text { ez } & \text { dituzte } & \text { beren } & \text { kanitak } & \text { hartu } \\ \text { ne/pas } & \text { ils/les/ont } & \text { leurs } & \text { les/couteaux } & \text { pris }\end{array}$

(10) zenbat idi dituzte herrian (Bidarray/Bidarrai, BN, EAEL 262 ) «combien de bœufs ont-ils dans leur village?»

$\begin{array}{llll}\text { zenbat } & \text { idi } & \text { dituzte } & \text { herrian } \\ \text { combien } & \text { bœuf } & \text { ils/les/ont } & \text { dans/le/village }\end{array}$




\section{Carte 4}

${ }^{*}$ Edun / eduki = haber / tener $\left(\right.$ EAEL $\left._{262}\right)$

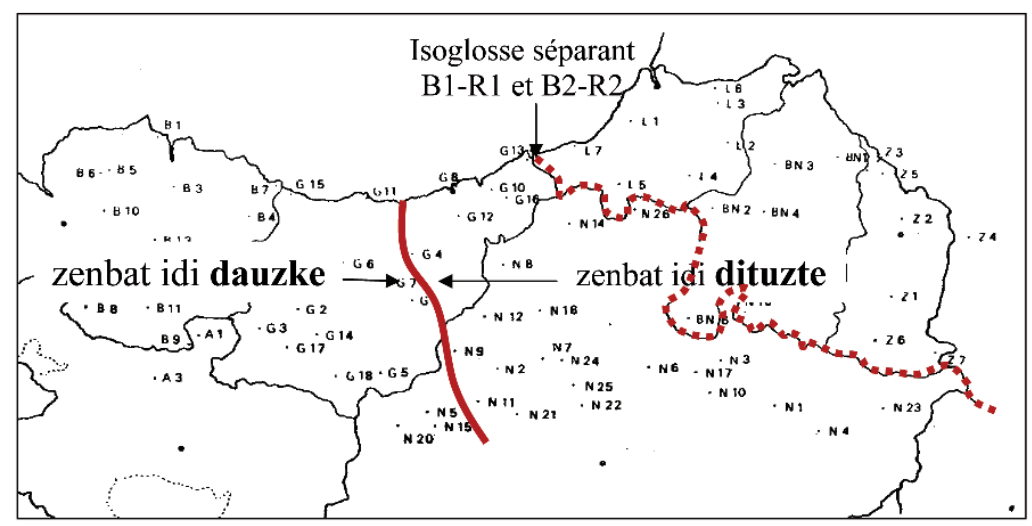

(i) Différence entre R1 et R2. Alors que le français utilise le verbe avoir à la fois comme auxiliaire et comme verbe à sens plein avec le sens de «posséder », l'espagnol dispose de deux verbes, haber et tener, le premier servant d'auxiliaire et le second convoyant le sens de «posséder ».

(ii) Vérification dans des corpus non traduits que la différence entre B1 et B2 préexiste à l'enquête. La vérification confirme que la différence entre B1 et B2 préexiste à l'enquête.

(iii) Jugement d'acceptabilité. Il n'est pas possible de tester la phrase (8) en B2 car le verbe eduki n'est pas utilisé sous cette forme par les informateurs. D'après mes informations, si l'on testait la phrase (10) dans l'aire Bl occidentale, on obtiendrait la réponse suivante $e^{15}$ :

\begin{tabular}{|c|c|c|c|c|}
\hline $\begin{array}{c}\text { Dans votre usage personnel de langue, } \\
\text { est-il possible de dire: }\end{array}$ & Oui & $\begin{array}{c}\text { Plutôt } \\
\text { oui }\end{array}$ & $\begin{array}{c}\text { Plutôt } \\
\text { non }\end{array}$ & Non \\
\hline $\begin{array}{c}\text { zenbat idi ditue errin? } \\
\text { « ¿cuántos bueyes tienen en el pueblo?» }\end{array}$ & & & & $\mathrm{X}$ \\
\hline
\end{tabular}

Le contraste dialectal entre B1 occidental et B2 serait donc en partie conditionné par le contraste dialectal entre R1 et R2, les informateurs de B1 occidental tendant à transposer dans leur langue l'opposition de l'espagnol haber / tener. Pour cela, il a été recouru au verbe autochtone eduki, verbe qui a été mis en opposition avec le verbe *edun équivalent de l'auxiliaire haber. Alors que eduki est utilisé (cf. espagnol tener) lorsqu'il s'agit de dénoter le sens de posséder, comme en (8), les mêmes informateurs utilisent *edun (cf. espagnol haber) comme auxiliaire comme en (7). Les locuteurs de B2 utilisent quant à eux *edun/ukan « avoir » dans les deux cas.

Le cas des phrases EAEL $L_{327}$ est intéressant car, tout en s'inscrivant dans la même configuration que celle qui vient d'être présentée, il montre qu'une étroite bande de l'aire latérale occidentale se dissocie de son aire adjacente et rejoint les parlers de l'aire orientale.

15. Prédiction à valider par enquête. 
(11) gose da (aires latérales, Alava, Biscaye ; L, BN, S, EAEL ${ }_{327}$ )

« il a faim / tiene hambre »

$\begin{array}{ll}\text { gose } & d a \\ \text { faim } & \text { il/être }\end{array}$

(12) gosea dauka (Aires centro-occidentale, B, G, EAEL ${ }_{327}$ )

$\begin{array}{ll}\text { gosea } & \text { dauka } \\ \text { el/hambre } & \text { él/la/tener }\end{array}$

Carte 5

Eduki $=$ haber $\left(\right.$ EAEL $\left._{327}\right)$

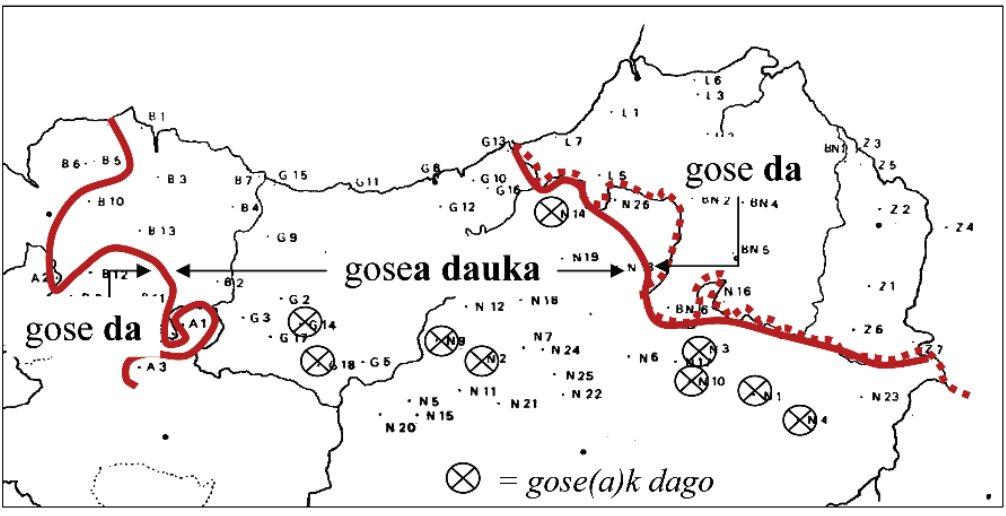

Je ne reprends pas les trois temps de la méthode puisqu'il se passe ici le même phénomène que celui qui vient d'être décrit. Il est néanmoins remarquable que l'aire latérale extrême-occidentale semble avoir appliqué le calque dans le cas de tener bueyes mais pas dans celui de tener hambre.

\section{Constructions obliques}

Observons les phrases et la carte suivantes:

(13) gizonak zakur bat yo du (Uhart-Cize/Uharte-Garazi, BN, EAEL ${ }_{241}$ )

«l'homme a frappé un chien»

$\begin{array}{lllll}\text { gizonak } & \text { txakur } & \text { bat- } \emptyset_{\mathrm{ABS}} & \text { jo } & d_{3 \mathrm{ABS}}-u_{\mathrm{AUX}}-\emptyset_{3 \mathrm{ERG}} \\ \text { l'homme } & \text { chien } & \text { un- } \emptyset & \text { frappé } & \text { le-avoir-il }\end{array}$

(14) gizonak yo du txakur bati (Iraberri, EAEL ${ }_{241}$ )

«el hombre a pegado a un perro»

$\begin{array}{lllll}\text { gizonak } & \text { jo } & \boldsymbol{d}_{\text {3ABS }}-\boldsymbol{u}_{\text {AUX }}-\emptyset_{\text {3ERG }} & \text { txakur } & \text { bat-i } \boldsymbol{i}_{\text {DAT }} \\ \text { el/hombre } & \text { pegado } & \text { le-haber-él } & \text { perro } & \text { un-a }\end{array}$

(15) gizonak zakur bati jo dio (Alli, N, EAEL ${ }_{241}$ )

«el hombre a pegado a un perro»

$\begin{array}{lllll}\text { gizonak } & \text { txakur } & \text { bat- } i_{\text {DAT }} & \text { jo } & d_{3 A B S}-i_{A U x}-o_{3 D A T}-\emptyset_{3 E R G} \\ \text { el/hombre } & \text { perro } & \text { un-a } & \text { pegado } & \emptyset \text {-haber-le-él }\end{array}$




\section{Carte 6}

\section{Constructions obliques (EAEL $\left.{ }_{241}\right)$}

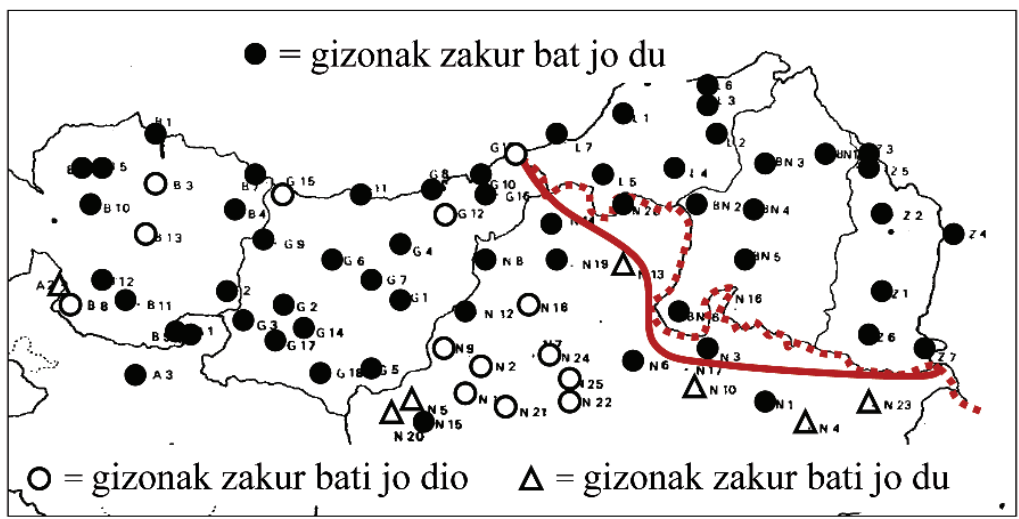

(i) Différence entre R1 et R2. L'espagnol fait précéder le complément animé de la préposition a (la cena está lista, llama a tu hermana) alors que le français ne le fait pas (le dîner est prêt, appelle ta søur). Or, comme on le voit, une majorité d'informateurs de B1 et tous ceux de B2 ont donné une phrase à auxiliaire bipersonnel mais un tiers des informateurs de B1 (et seulement de B1) ont donné un syntagme totalement ou partiellement oblique (usage homologue à celui de l'espagnol dans ce contexte).

(ii) Vérification dans des corpus non traduits que la différence entre B1 et B2 préexiste à l'enquête. La vérification confirme que la différence entre B1 et B2 préexiste à l'enquête.

(iii) Jugement d'acceptabilité. Évaluation fournie par des informateurs de Soule:

\begin{tabular}{|c|c|c|c|c|}
\hline $\begin{array}{c}\text { Dans votre usage personnel de langue, } \\
\text { est-il possible de dire : }\end{array}$ & Oui & $\begin{array}{c}\text { Plutôt } \\
\text { oui }\end{array}$ & $\begin{array}{c}\text { Plutôt } \\
\text { non }\end{array}$ & Non \\
\hline $\begin{array}{c}\text { gizonak tzakür bat jo dü } \\
\text { «'homme a frappé un chien » } \\
\text { (déterminant à l'absolutif + auxiliaire bipersonnel) }\end{array}$ & $\mathrm{X}$ & & & \\
\hline $\begin{array}{c}\text { gizonak tzakür bati jo dü } \\
\text { (déterminant au datif + auxiliaire bipersonnel) }\end{array}$ & & & & $\mathrm{X}$ \\
\hline $\begin{array}{c}\text { gizonak tzakür bati jo deio } \\
\text { (déterminant au datif + auxiliaire tripersonnel) }\end{array}$ & & & & $\mathrm{X}$ \\
\hline
\end{tabular}


Il serait ici intéressant de soumettre le questionnaire suivant aux informateurs de B1:

\begin{tabular}{|c|c|c|c|c|}
\hline $\begin{array}{c}\text { Dans votre usage personnel de langue, } \\
\text { est-il possible de dire : }\end{array}$ & Oui & $\begin{array}{c}\text { Plutôt } \\
\text { oui }\end{array}$ & $\begin{array}{c}\text { Plutôt } \\
\text { non }\end{array}$ & Non \\
\hline $\begin{array}{c}\text { gizonak zakur bat jo du } \\
\text { «el hombre a pegado a un perro » } \\
\text { (déterminant à l'absolutif + auxiliaire bipersonnel) }\end{array}$ & & & & \\
\hline $\begin{array}{c}\text { gizonak zakur bati jo du } \\
\text { (déterminant au datif + auxiliaire bipersonnel) }\end{array}$ & & & & \\
\hline $\begin{array}{c}\text { gizonak zakur bati jo dio } \\
\text { (déterminant au datif + auxiliaire tripersonnel) }\end{array}$ & & & & \\
\hline
\end{tabular}

Quelques précisions pour conclure.

Le fait qu'une isoglosse séparant deux traits dialectaux, en basque, coïncide avec l'isoglosse séparant le domaine hispano-roman du domaine gallo-roman n'implique pas forcément que les traits basques considérés s'expliquent par un conditionnement roman. Inversement, le fait qu'une isoglosse séparant deux traits dialectaux, en basque, soit disjointe de l'isoglosse séparant le domaine hispano-roman du domaine gallo-roman, n'implique pas forcément que les traits basques considérés n’ont pas subi un conditionnement roman.

Les tests d'admissibilité se révèlent être une expérience à la fois très instructive et d'un maniement parfois délicat. Ne pouvant ici entrer dans le détail, citons simplement les quelques aspects suivants. Les informateurs ont jugé l'éventail de réponses proposé (« oui »; « plutôt oui »; « plutôt non »; «non ») comme adapté et suffisamment souple. La réponse « plutôt non » n'ayant presque jamais été renseignée, une tripartition « oui »; " plutôt oui »; «non » semblerait suffisante dans le cadre d'une première approche. Laspect délicat de ce type d'enquête tient à la nature même des informations recherchées, qui font appel à des ressentis évaluatifs et comportent donc un aspect subjectif ${ }^{16}$.

16. Les informateurs n'ont pas de mal à se déterminer dans beaucoup de cas. Mais dans certains cas, le jugement d'acceptabilité peut s'avérer difficile à établir. Le problème est connu de la même façon lorsque l'on teste des possibles de langue dans des langues fortement standardisées comme le français, y compris avec des informateurs expérimentés. 
Pour un aperçu de l'évolutivité diachronique de certains des faits évoqués dans la note, on se reportera notamment au $D G V^{17}$. On peut penser que l'étude diachronique s'accordera dans certains cas avec les données contemporaines pour étayer l'hypothèse du calque et que, dans d'autres cas, elle ne pourra apporter d'éléments décisifs ni dans un sens ni dans un autre.

\section{Indications bibliographiques}

Alarcos Llorach, Emilio, 1994, Estudios de gramática funcional del español, Madrid, Gredos. [1970]

Allières, Jacques, 1979, Manuel pratique de basque, Paris, Picard.

Bosque Ignacio \& Demonte Violeta (dir.), 2000, Gramática descriptiva de la lengua española (3 vol.), Madrid, Espasa.

Brunot Ferdinand \& Bruneau Charles, 1969, Précis de grammaire historique de la langue française, Paris, Masson et $\mathrm{c}^{\text {ie }}$.

Caron Philippe \& Liu Yu-Chang, 1999, « Nouvelles données sur la concurrence du passé simple et du passé composé dans la littérature épistolaire », Linformation grammaticale n 82, juin 1999 , pp. 39-50.

Leizaola Fermín (dir.), 1983-1990, Euskal Herriko Atlas Etnolinguistikoa (EAEL), Donostia, Aranzadi zientzia elkartea.

Michelena Luis \& Sarasola Ibon (dir.), 1987, Diccionario General Vasco (DGV)/Orotariko Euskal Hiztegia, Bilbao/Bilbo, Euskaltzaindia.

Orpustan Jean-Baptiste, 1997, Basque et français. Méthode abrégée de traduction, Baigorri, Izpegi.

Rohlfs Gerhard, 1977, Le gascon. Études de philologie pyrénéenne, Halle, Max Niemeyer Verlag-Pau, Marrimpouey Jeune. [1935]

Trask, Robert Lawrence, 1997, The history of Basque, London-New York, Routledge.

Yllera Alicia, 1980, Sintaxis histórica del verbo español: las perífrasis medievales, Zaragoza, Departamento de Filología francesa de la universidad de Zaragoza.

Zuazo Koldo, 2003, Euskalkiak: Herriaren lekukoak, Donostia, Elkar.

\section{(Footnotes)}

1. La phrase testée doit être compatible avec le parler des intéressés. Ici c'est le temps de l'auxiliaire qui est testé (zen = passé), pas le participe (etorri n'est pas utilisé dans le dialecte).

2. Le verbe egon synthétique au passé n'est pas utilisé par les informateurs, mais egoiten zen n'est pas non plus jugé possible dans le contexte de la phrase source.

17. Izan / egon = ser / estar : DGV VI, p. 446. *Edun / eduki = haber / tener : DGV VI, p. 384 et p. 396. Constructions obliques: DGV VI, p. 77 et DGV X, p. 232. (Cf. aussi entre autres: Trask, 1997 : 292-293, etc.) Pour les usages diachroniques comparatifs du passé simple et passé composé en français, cf. Caron \& Liu (1999). 